\title{
Extensive Survey on MIMO Technology using V-BLAST Detection Technique
}

\author{
Sudhanshu Kumar Chourasia \\ Department of Electronics \\ \& Communication Engineering \\ Vedica Institute of Technology, Bhopal
}

\author{
Rashmi Pandey \\ Department of Electronics \\ \& Communication Engineering \\ Vedica Institute of Technology, Bhopal
}

\begin{abstract}
Wireless communication using Multiple Input Multiple Output (MIMO) systems increases efficiency for a given total transmits power. Here investigate a novel approach to perform decoding- order optimization in the vertical Bell Labs layered space-time (V-BLAST) planning. Allowing for instantaneous transmission of multiple, independently encoded data streams, the V-BLAST transmit-receive method applies to the uplink of a multi-user communication system. A multi-antenna receiver decodes data streams consecutively, trade with inter-stream interference by means of cancelling and nulling; interference from before decoded streams is cancelled by subtraction, and residual interference is nulled by linear equalization. The order in which the streams are decoded can have a visible impact on system presentation. Multiple Input Multiple Output (MIMO) channels can offer high capability to wireless systems and the capacity increases linearly with the number of antennas. There are many schemes that can be applied to MIMO systems such as space time block codes, space time trellis codes, and the Vertical Bell Labs Space-Time structural design (V-BLAST). We study the broad-spectrum MIMO system, the general VBLAST structure, and the Successive Interference Cancellation (SIC) Zero-Forcing (ZF) detectors in this paper.
\end{abstract}

\section{Index Terms}

Communication systems, V-BLAST, ZF, successive interference cancellation, decision feedback equalizers, fading channels.

\section{INTRODUCTION}

Latest research on wireless communication systems has shown that using multiple antennas at both transmitter and receiver offer the possibility of wireless communication at higher data rates compared to single antenna systems. The informationtheoretic capacity of these multiple-input multiple-output (MIMO) channels was exposed to grow linearly with the minor of the numbers of transmit and receiver antennas in rich scattering environments, and at sufficiently high signal-to-noise (SNR) ratios [1]. Some unique detection algorithms have been planned in order to exploit the high spectral capacity offered by MIMO channels. One of them is the V-BLAST (Vertical BellLabs Layered Space-Time) algorithm which uses a layered structure [2]. This algorithm offers extremely better error performance than conventional linear receivers and still has low complexity. In the history, theoretical research on multipleinput multiple-output (MIMO) systems that use multiple transmit and receive antennas in a rich scattering communication channel have provided luminous results. They have given a linear increase in system ability and spectral efficiency with respect to the number of transmit antennas as long as the number of receive antennas is better or equal to the number of transmit antennas [3], [4]. In a MIMO communication system, multiple transmission paths can be used to get better diversity and or multiplexing gain. In the VBLAST transmitter, each antenna transmits its own separately coded symbols and the V-BLAST receiver, uses a spatial area decision feedback equalizer. The process involves each symbol is decoded and then fed back to cancel its interference with extra symbols. This process repeats until all the symbols are decoded. The decoding order can be optimized by decoding the symbol with largest signal to noise ratio (SNR) first. Due to this decision feedback structure, the V-BLAST system with MIMO technology yields a very good spectral efficiency in a scattering rich environment. The reimbursement is attainable without increasing the transmission bandwidth or power.

\section{MIMO CHANNEL}

Multiple input multiple-output (MIMO) systems are a usual addition of developments in antenna array communication. At the same time as the advantages of multiple receive antennas, such as gain and spatial diversity, have been recognized and exploited for several time, the use of transmit diversity has only been investigated lately. The advantages of MIMO communication, which exploits the physical channel between several transmit and receive antennas, are now receiving significant concentration. Techniques that employ arrays of multiple transmit and receive antennas may suggest high capacity to present and future wireless communications systems, which put severe difficulty on current spectral resources. MIMO systems offer a number of advantages over single-antenna-to-single-antenna communication. Sensitivity to fading is concentrated by the spatial diversity provided by multiple spatial paths. Underneath certain environmental conditions, the power necessities connected with high spectraleffectiveness communication can be significantly reduced by avoiding the compressive area of the information-theoretic ability bound. Here, spectral efficiency is defined as the total number of information bits per second per Hertz transmitted as of one array to the other.

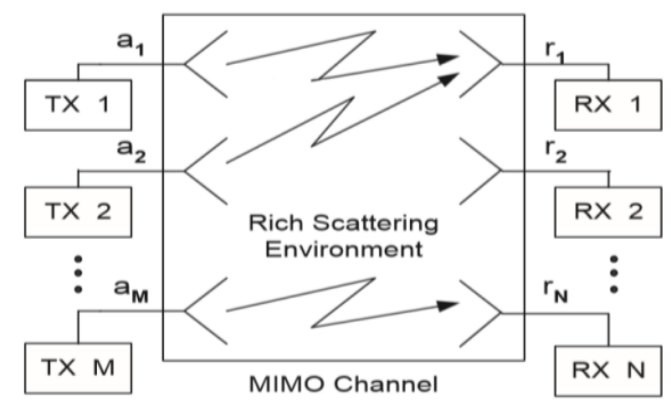

Figure 1: Multiple Input Multiple Output (MIMO) channel model 
Multiple-input multiple-output (MIMO) systems offer for a linear increase of capacity with the number of antenna elements, affording significant increases in excess of singleinput single-output (SISO) systems. To assess the performance of MIMO systems, the MIMO channel must be suitably modeled. It is frequent to model the MIMO channel assuming an independent quasi-static flat Rayleigh fading at all antenna workings. There are various schemes that can be applied to MIMO systems such as space time block codes [5] [6], space time trellis codes [7] and Bell-Labs Layered Space Time architecture [8]. With a simple MIMO channel system consisting of $T \mathrm{n}$ transmit antennas and $\mathrm{R} n$ receive antennas, the channel matrix is described as

$$
H=\left(\begin{array}{ccc}
h_{11} & \ldots & h_{1 n_{T}} \\
\vdots & \ddots & \vdots \\
h_{n_{R} 1} & \cdots & h_{n_{R} n_{T}}
\end{array}\right)
$$

Where

$$
\begin{aligned}
h_{i j}= & \alpha+j \beta \\
= & \sqrt{\alpha^{2}+\beta^{2}} e^{-j \arctan \frac{\beta}{\alpha}} \\
& h_{i j}\left|e^{j \varphi i j}\right|
\end{aligned}
$$

In a rich scattering background without line-of-sight, the path gains as exposed in Figure 2 from $\mathrm{j}$ transmit antenna to $\mathrm{i}$ receive antenna are Rayleigh scattered. These path gains are modeled with zero mean and 0.5 variance independent complex Gaussian random variables per measurement. The channel individuality is not changed throughout the transmission period of a whole frame in accordance with the quasi-static flat fading statement. The spatial separation of antenna elements is sufficient to lead to independent channel outcome.

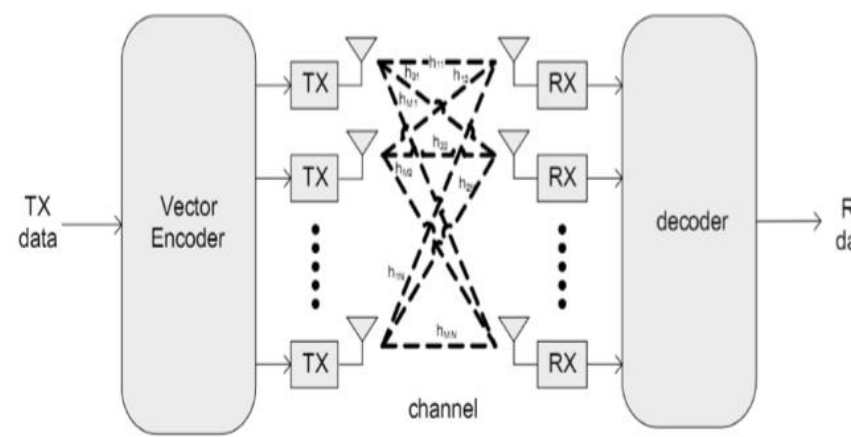

Figure 2: Graphic of MIMO channel

\section{DIFFERENT RECEIVER DETECTION TECHNIQUES}

The V-BLAST detection method adopted for MIMO are classified as Linear and Non Linear detection schemes. Beneath Linear technique we have MMSE and ZF. Where as in Non linear we have ML. They are described as follows:

\section{A. Maximum Likelihood (ML)}

ML, Non Linear detection that contrasts the accepted indicators and all conceivable transmitted sign vectors. This is changed by channel grid $\mathrm{H}$ and assessments transmit image vector " $x$ " as per the most extreme Probability Guideline. The fundamental detriment of ML finder is that, it gets to be restrictively unpredictable due to its unpredictability which builds exponentially with the transmitter radio wire number and the balance request [9].

$$
\text { Xnew }=\arg x k \epsilon\{x 1, x 2 \ldots x n\} \min \|p-H X k\|^{2}
$$

Where $\mathrm{p}$ is the received symbol, $\mathrm{x}_{\mathrm{k}}$ is the transmitted symbol at the $\mathrm{k}^{\text {th }}$ iteration.

\section{B. Zero Forcing $(Z F)$ :}

By utilizing straight location procedure, at the collector frontclosures to partitioned the transmitted information streams, and afterward autonomously translate each of the streams. Basic direct beneficiary with low computational unpredictability and experiences clamor improvement. It works best with high SNR.

$$
\text { xnew }=\left(\left(H^{T} H\right)^{-1} \mathrm{H}\right) * \mathrm{X}
$$

Where $\mathrm{H}^{\mathrm{T}}$ is the transpose of channel matrix $\mathrm{H}$.

\section{Minimum Mean Square Error (MMSE):}

The MMSE recipient smothers both the impedance and clamor segments. This intimates that the mean square failure between the transmitted images and the evaluation of the beneficiary is minimized. The vital gimmick of MMSE locators are that, they are better analyzed than ZF. At Low SNR, MMSE gets matched channel. Also at high SNR, MMSE becomes ZeroForcing.

$$
\text { Xnew } \left.=\text { D. } x=\left(\left(\frac{1}{S N R}\right) I N_{r}+H^{H} H\right)\right)^{-1} \cdot \mathrm{H}^{\mathrm{H}} \mathrm{X}
$$

Where D is a channel variable as a function of $\mathrm{I}_{\mathrm{Nr}}$. Here $\mathrm{I}_{\mathrm{Nr}}$ is the information of the symbol ' $\mathrm{x}$ ' received at the $\mathrm{N}^{\text {th }}$ iteration and $\mathrm{H}^{\mathrm{H}}$ is the projection of channel matrix $\mathrm{H}$.

\section{QR Decomposition:}

Given a matrix A, its QR decomposition is a matrix decomposition of the form

$$
A=Q R
$$

Where $\mathrm{R}$ is an upper triangular matrix and $\mathrm{R}$ is an orthogonal matrix, i.e., one satisfying

$$
Q^{T} \cdot Q=I
$$

Where $\mathrm{Q}^{\mathrm{T}}$ is the transpose of $\mathrm{Q}$ and $\mathrm{I}$ is the identity matrix.

In V-BLAST, the amount of Transmit Receiving wires ought to be short of what number of gets reception apparatuses. The images could be transmitted with coding or without coding i.e., regulation. The location procedure comprises of two fundamental operations:

a). Interference Suppression (Nulling): The concealment operation nulls out obstruction by anticipating the gained vector onto the invalid subspace (perpendicular subspace) of the subspace spread over by the meddling signs. After that, ordinary identification of the first image is performed [10]

b). Interference cancellation (subtraction): For SIC: The commitment of the distinguished image is subtracted from the gained vector. For PIC: This discovery procedure is to recover all the while after balance all the meddling images focused around past estimations [11]

\section{LITERATURE REVIEW}

Gurpreet Singh, Rahul Vij and Priyanka Mishra [12] Wireless communication technology has shown that the applying of multiple antennas at each transmitter and receiver sides improve the chance of high knowledge rates through knowledge rates through multiplexing or to boost performance through diversity compared to single antenna systems. during this article, we have a tendency to studied the BER 
performance of Vertical Bells lab layered space Time design (V-BLAST) spatial Multiplexing Technique with numerous decoding techniques like maximum likelihood (ML), Minimum Mean square Error (MMSE), Minimum Mean sq. Error + Ordered Serial Interference Cancellation (MMSE+OSIC), MMSE, Zero Forcing, Zero Forcing + Ordered Serial Interference Cancellation (ZF+OSIC) by victimization completely different modulation techniques like BPSK, QPSK, 16-QAM in independent, identically distributed flat attenuation channel.

Joshi, S.A., Rukmini, T.S. ; Mahesh, H.M. [13], This paper proposes indicator identifiers for V-Impact construction modeling with Maximum Likelihood (ML), Zero Forcing (ZF), Minimum Mean-Square Error (MMSE), and Successive Interference Cancellation (SIC) finders and reproduces these structures in Rayleigh blurring channel. The proposed investigation additionally contrasts the exhibitions of MIMO framework and diverse balance systems like BPSK and QPSK in Blurring and AWGN channels. In view of bit slip rates, we examine the execution and the computational many-sided quality of these plans.

Shreedhar. A. Joshi, Dr. Rukmini T S, Dr. Mahesh H M [14], The V-BLAST (Vertically - layered Bell Laboratories Layered Space-Time) calculation is a multi- layer image recognition plan. The proposed work concentrates on V-Impact procedure with a Multiple Input Multiple output (MIMO) engineering emulated by recipient identification methods like forcing (ZF), Minimum Mean Square Error (MMSE) with back substitution SIC (Symbol Interference Cancellation). The proposed approach embraces BPSK, QAM regulation routines. The numerical investigation is directed utilizing MATLAB. The execution change is additionally noteworthy and Reenactment results indicate that V-Impact accomplishes better Bit slip rates.

Kai Wu, Lin Sang, He Wang, Cong Xiong, Dacheng Yang, Xin Zhang [15], In the customary zero-forcing ordered successive interference cancellation (ZF-OSIC) or minimum mean square error ordered successive interference cancellation (MMSEOSIC) identification calculation of vertical Bell-Labs layered space-time (V- BLAST) architecture, there exists an undesirable handling defer because of the count of the framework pseudo-converse and force reordering in recognizing the image in each one layer. The parallel interference cancellation (PIC) algorithm has a more level preparing postpone yet poorer execution. In view of the peculiarities of SIC and PIC calculations, a coordinated PIC and OSIC detection (IPOD) calculation is proposed which can bring down the multifaceted nature and the preparing deferral of the entire calculation with little execution misfortune. In addition, there is an alterable parameter in IPOD calculation which could be changed to acquire distinctive tradeoff between unpredictability and execution. In this manner, the proposed calculation is more doable for reasonable provisions.

Wu Nian Wang Zhongpeng Zhang Shaozhong [16], in this paper, a few recognition calculations focused around MIMOOFDM frameworks are quickly presented, and their exhibitions are assessed by workstation recreation. The reproduction results show that execution of these calculations for MIMOOFDM framework is like the execution for level MIMO framework. We reason that the so- called ZF-PIC calculation is exceptionally appealing contrasted with traditional ZFVBLAST in execution.

Jiming Chen, Shan Jin, and Yonggang Wang [17], Hypothetical and exploratory studies have demonstrated that layered space-time architectures like the Vertical Ringer labs Layered Space-Time (V-Blast) framework can misuse the limit point of interest of numerous receiving wire frameworks in rich-disseminating situations. In this paper, we introduce a decreased multifaceted nature calculation for identifying such structural engineering regarding the Minimum Mean Square Error (MMSE) paradigm. This calculation bases on the tried and true SIC identification calculation, yet chooses a few layers with sufficiently substantial indicator to impedance in addition to clamor degree (SINRs) rather than the layer with biggest SINR at each one phase of progressive abrogation, and uses GSO to substitute the processing of pseudo-opposite in discovering the weight vectors. Subsequently the computational many-sided quality of the proposed recognition calculation is altogether diminished however the execution debasement is little.

Heunchul Lee, Byeongsi Lee and Inkyu Lee [18], in this paper, we present an enhanced vertical Ringer Labs layered spacetime (V-Impact) recipient which considers the choice slips. Second, we propose an iterative detection and decoding (IDD) plan for coded layered space-time architectures in MIMOOFDM frameworks. For the iterative process, a low- manysided quality demapper is created by making utilization of both non- direct obstruction abrogation and straight least meansquare lapse filtering. Likewise, a straightforward dropping technique focused around hard choice is introduced to diminish the general multifaceted nature. Recreation results exhibit that the proposed IDD plan joined together with the enhanced $\mathrm{V}$ Impact performs just about and the ideal turbo-MIMO methodology, while giving huge funds in computational unpredictable.

Table 1: Summary of Literature Review

\begin{tabular}{|c|c|c|c|c|}
\hline Year & Author & Title & Approach & Result \\
\hline 2012 & $\begin{array}{l}\text { Gurpreet Singh, Rahul } \\
\text { Vij and Priyanka } \\
\text { Mishra }\end{array}$ & $\begin{array}{l}\text { Performance Evaluation for } \\
\text { V-Blast MIMO Systems } \\
\text { under Various Modulation } \\
\text { Schemes Using Ricean } \\
\text { Channel }\end{array}$ & $\begin{array}{l}\text { Spatial Multiplexing Technique } \\
\text { with various decoding techniques }\end{array}$ & $\begin{array}{l}\text { Got more optimal result } \\
\text { for } 1 \times 4 \\
\text { antennas }\end{array}$ \\
\hline 2011 & $\begin{array}{l}\text { Joshi, S.A., Rukmini, } \\
\text { T.S. ; Mahesh, H.M. }\end{array}$ & $\begin{array}{l}\text { Error rate analysis of the V- } \\
\text { BLAST MIMO channels } \\
\text { using interference } \\
\text { cancellation detectors }\end{array}$ & $\begin{array}{l}\text { V-BLAST architecture with } \\
\text { Maximum Likelihood (ML), Zero } \\
\text { Forcing (ZF), Minimum Mean- } \\
\text { Square Error (MMSE), and } \\
\text { Successive Interference } \\
\text { Cancellation (SIC) }\end{array}$ & $\begin{array}{l}\text { Analyze the performance } \\
\text { of bit error rates and } \\
\text { computational } \\
\text { complexity }\end{array}$ \\
\hline 2010 & $\begin{array}{l}\text { Shreedhar. A. Joshi, } \\
\text { Dr. Rukmini T S , Dr. } \\
\text { Mahesh H M }\end{array}$ & $\begin{array}{l}\text { Performance Analysis of } \\
\text { MIMO Technology using V- } \\
\text { BLAST Technique for }\end{array}$ & $\begin{array}{l}\text { V-BLAST technique with a } \\
\text { Multiple Input Multiple output } \\
\text { (MIMO) technology }\end{array}$ & $\begin{array}{c}\text { Achieves better Bit error } \\
\text { rates }\end{array}$ \\
\hline
\end{tabular}




\begin{tabular}{|c|c|c|c|c|}
\hline \multirow{2}{*}{2009} & $\begin{array}{c}\text { Different Linear Detectors in } \\
\text { a Slow Fading Channel } \\
\text { Kai Wu, Lin Sang, He } \\
\text { Wang, Cong Xiong, } \\
\text { Dacheng Yang, Xin } \\
\text { Zhang }\end{array}$ & $\begin{array}{c}\text { Detection Algorithm for V- } \\
\text { BLAST Systems with Novel } \\
\text { Interference Cancellation } \\
\text { Technique }\end{array}$ & $\begin{array}{c}\text { Integrated PIC and OSIC } \\
\text { detection (IPOD) algorithm }\end{array}$ & Lower the complexity \\
\hline \multirow{2}{*}{2008} & $\begin{array}{c}\text { Wu Nian Wang } \\
\text { Zhongpeng Zhang } \\
\text { Shaozhong }\end{array}$ & $\begin{array}{c}\text { Performance Comparison of } \\
\text { Sub-optimal Algorithms } \\
\text { Based on MIMO-OFDM } \\
\text { Systems }\end{array}$ & $\begin{array}{c}\text { Detection algorithms based on } \\
\text { MIMO-OFDM systems }\end{array}$ & Improved performances \\
\hline \multirow{2}{*}{2006} & $\begin{array}{c}\text { Jiming Chen, Shan Jin, } \\
\text { and Yonggang Wang, }\end{array}$ & $\begin{array}{c}\text { Reduced Complexity } \\
\text { MMSE-SIC Detector in V- } \\
\text { BLAST Systems }\end{array}$ & $\begin{array}{c}\text { Successive Interference } \\
\text { Cancellation (SIC) Detection } \\
\text { algorithm }\end{array}$ & $\begin{array}{c}\text { Significantly reduced the } \\
\text { performance }\end{array}$ \\
\hline & $\begin{array}{c}\text { Heunchul Lee, } \\
\text { Inteongsi Lee and }\end{array}$ & $\begin{array}{c}\text { Iterative Detection and } \\
\text { Decoding With an Improved } \\
\text { V-BLAST for MIMO- } \\
\text { OFDM Systems }\end{array}$ & $\begin{array}{c}\text { Iterative detection and decoding } \\
\text { (IDD) }\end{array}$ & $\begin{array}{c}\text { Reduced computational } \\
\text { complexity }\end{array}$ \\
\hline
\end{tabular}

\section{CONCLUSION}

In this paper, a general idea of MIMO with the V- Blast procedure utilizing a few indicators (MMSE, ML, ZF and QR) is introduced. A near investigation of different direct and non straight finders is indicated. Also indicate the profits of requesting methodology over SIC and PIC abrogation techniques. MIMO is a vital engineering for empowering the remote business to convey an immense potential and guarantee of remote broadband. Nonetheless, the disadvantage of Impact calculations is the spread of choice blunders. Besides, because of the impedance concealment, early identified images at the recipient regale from more level differing qualities than later ones. Subsequently, the calculation brings about unequal differing qualities advantage for every image. In this paper, display an asymptotic examination of the VBLAST plan at high SNR area. Both the ZF -V-Blast and MMSE-V -Blast with SIC are investigated regarding their differences additions and BER performance.

\section{ACKNOWLEDGMENT}

At the outset I would like to express my deep sense of gratitude to my respected dissertation guide and HOD, under whose valuable guidance, my project saw the light of the day. I will be ever indebted to her for his morale boosting support \& encouragement.

\section{REFERENCES}

[1] I. E. Telatar, "Capacity of multi-antenna Gaussian channels," Eur. Trans. Tel., vol. 10, pp. 585-595, November-December 1999.

[2] P. W. Wolniansky, G. J. Foschini, G. D. Golden, and R. A. Valenzuela, "V-blast: An architecture for realizing very high date rates over the rich- scattering wireless channel," Proc. URSI ISSSE, pp. 295-300, 1998.

[3] G. J. Foschini and M. J. Gans, "On the limits of wireless communications in a fading environment when using multiple antennas," Wireless Personal Communications, vol 6, no. 3, pp. 311-335, 1998.

[4] G. J. Foschini and R. A. Valenzuela: "Initial estimation of communications efficiency of indoor wireless channels," Wireless Networks, vol. 3, no. 2, pp. 141-154, 1997.

[5] S. M. Alamouti, "A simple transmit diversity technique for wireless communications," IEEE Journal on Selected
Areas in Communications, vol.16, pp. 1451 1458, October 1998

[6] V. Tarokh, H. Jafarkhani, A. R. Calderbank, "Space-Time Block Coding for wireless communications: Performance Results," IEEE Journal on Selected Areas in Communications, vol.17, pp.451 460, March 99.

[7] V.Tarokh, N. Sesahdri, A. R. Calderbank, "Space-Time codes for high data rate wireless communication: Performance criterion and code construction," IEEE Transactions on Information Theory, vol.44, pp.744 765, March 1998.

[8] Gerard J. Foschini, "Layerd space-time architecture for wireless communications in a fading environment when using multi-element antennas," Bell Labs Technical Journal, autumn 1996.

[9] Nirmalendu Bikas Sinha, R. Bera, M. Mitra : "Capacity and V-BLAST techniques for MIMO wireless channel". Journal of Theoretical and Applied Information Technology @ 2005 - 2010 JATIT.

[10] Shreedhar. A. Joshi, Dr. Rukmini T S, Dr. Mahesh H M. "Performance analysis of MIMO Technology using VBLAST Technique for different linear Detectors in a slow fading channel." IEEE International Conference on Computational conference on Computational Intelligence and Computing Research (ICCIC'2010).978-1-42245966-7/10. P 453-456.

[11] Zhanyun Duan, Tobias Hidalgo Stitz, Mikko Valkama, and Markku Renfors "Performance Analysis of Parallel Interference Cancellation Detector in Downlink MCCDMA Systems", Proceedings of the 6th Nordic Signal Processing Symposium-NORSIG 2004 June 9-11,2004 Espoo, Finland.

[12] Gurpreet Singh, Rahul Vij and Priyanka Mishra, "Performance Evaluation for V-Blast MIMO Systems under Various Modulation Schemes Using Ricean Channel" IOSR Journal of Engineering (IOSRJEN) ISSN: 2250-3021 Volume 2, Issue 8 (August 2012), PP 201-210

[13] Joshi, S.A., Rukmini, T.S. ; Mahesh, H.M., "Error rate analysis of the V-BLAST MIMO channels using interference cancellation detectors" International Conference on Signal Processing, Communication, Computing and Networking Technologies (ICSCCN), 2011 IEEE 
[14] Shreedhar. A. Joshi , Dr. Rukmini T S , Dr. Mahesh H M, "Performance Analysis of MIMO Technology using VBLAST Technique for Different Linear Detectors in a Slow Fading Channel" 2010 IEEE.

[15] Kai Wu, Lin Sang, He Wang, Cong Xiong, Dacheng Yang, Xin Zhang, "Detection Algorithm for V-BLAST Systems with Novel Interference Cancellation Technique" 2009 IEEE

[16] Wu Nian Wang Zhongpeng Zhang Shaozhong, "Performance Comparison of Sub-optimal Algorithms Based on MIMO-OFDM Systems" 2008 IEEE international conference on communication technology proceeding.

[17] Jiming Chen, Shan Jin, and Yonggang Wang, "Reduced Complexity MMSE-SIC Detector in V-BLAST Systems" The 18th Annual IEEE International Symposium on Personal, Indoor and Mobile Radio Communications (PIMRC'07).

[18] Heunchul Lee, Byeongsi Lee and Inkyu Lee, "Iterative Detection and Decoding With an Improved V-BLAST for MIMO-OFDM Systems" IEEE Journal on Selected Areas in Communications, Vol. 24, No. 3, March 2006 